\title{
LINEAR PROCTOTOMY.
}

\author{
By CHARLES B. BALL, M.CH., F.R.C.S.;
}

University Examiner in Surgery; Surgeon to Sir Patrick Dun's Hospital.

[Read in the Section of Surgery, June 7, 1889.]

LINEAR proctotomy or external rectisection is an operation which has not, I feel convinced, met with the support from the profession in the United Kingdom which it merits ; and except as a preliminary to amputation of the rectum is but seldom had recourse to.

It may be defined as the making a deep incision through the anus and rectum directly backwards to the coccyx and hollow of the sacrum, and the following are probably the chief indications for its performance:-

I. For the cure of non-malignant strictures of the rectum in which gradual dilatation by means of bougies, or other instruments, is either impracticable or inefficient.

II. For the removal of new growths situated high up in the bowel or in the hollow of the sacrum.

III. As a palliative measure, instead of colotomy, to relieve obstruction in advanced malignant disease.

IV. As a preliminary step to amputation of the rectum.

The treatment of rectal stricture by bougies, mechanical dilators, or expansile bags, is, in many instances, eminently unsatisfactory; and we occasionally meet with cases in which it is impossible to dilate the constriction to any appreciable extent, consistent with the avoidance of rupture, while in other instances the bougies give rise to so much irritation that their use has to be discontinued; so that in order to overcome the difficulty something more is required, and the operations which have been practised with this view are-internal section, linear proctotomy, excision, and colotomy. Internal section, by means of a guarded, blunt pointed knife, has met with but little support, and it is open to serious objections. 
In the first place-if the incision, or incisions, are confined to the thick ring of indurated tissue constituting the stricture, but little relief is afforded, owing to the tough and resisting nature of the structures; while, if they extend into the healthy tissue outside, fæcal extravasation and stercoral abscess will be the necessary result. In this respect the treatment of rectal strictures offers a marked contrast to a similar condition in the urethra; in the latter the treatment by internal incision is probably the most favoured operation, and we know that it but rarely is followed by urinary extravasation or urethral abscess; the cause of this difference is not far to seek. When the urine is enabled to pass the strictured urethra by means of the incision it escapes freely, as it has no further obstruction to overcome; and in passing out at the meatus it merely follows the course of least resistance; but in the rectum the fæcal mass, after passing the stricture, has to distend the anus before it can escape, and a considerable amount of pressure is necessary for this purpose. In the effort to accomplish this some frces will be extravasated, to a certainty, if the internal incision has penetrated through the rectal wall. When, however, linear proctotomy has been performed, and the incision carried, not only through the entire stricture, but also through the lower portion of the rectum and anus, no resistance is offered to the fæcal mass, and extravasation is an impossibility. Excision of non-malignant strictures can scarcely be defended, as any cases situated low enough down to be effectually dealt with in this way can be admirably treated by linear proctotomy. Where, however, the stricture extends very high up, colotomy must still be looked upon as the only resource.

As it has frequently happened that fistulæ in connection with stricture have been operated upon without the stricture being diagnosed, and as the internal openings of these fistula are occasionally situated above the stricture, it is evident that complete division of the contraction has been thus sometimes unintentionally performed; but the first deliberate attempt to cure stricture in this way appears to have been made by Mr. Humphry, of Cambridge,

\footnotetext{
association Medical Journal, p. 21, 1856.
} 
who proposed this method in consequence of the "good results following longitudinal incisions in urethral stricture." And although he performed the operation on two occasions with excellent results, this method of treatment appears to have fallen into disuse until comparatively recently. To M. Verneuil is undoubtedly due the credit of having revived this operation, and of having brought it prominently before the profession. In a paper read at the Surgical Society of Paris, ${ }^{a}$ he enters very fully into the subject, and enumerates ten cases of the operation.

Can we anticipate a complete and absolute cure by this treatment in cases of non-malignant stenosis? To this question I am convinced that the answer can be given in the affirmative. If the operation has been completely performed, that is, if the entire thickness of indurated structures has been divided, a permanent cure will result in the majority of instances, and, as compared with colotomy, the relative mortality is slight. After the recovery of the patient the power of controlling the evacuations is generally fairly well regained, so that the occurrence of incontinence is improbable. The operation may be performed by different methods. Verneuil recommends the following plan:-The bowel having been well cleared out, and the patient placed in the lithotrmy position, the finger is introduced through the stricture. If difficulty is experienced in doing this, a probe-pointed bistoury is first passed, and an incision sufficient to allow of the easy introduction of the finger is made in a direction directly backwards. A trocar and cannula is now entered at the tip of the coccyx, and pushed on till it enters the rectum well above the seat of stricture. A flexible bougie, or piece of string, is by means of the cannula passed into the rectum, the end being hooked out at the anus with the finger. By means of this the chain of an écraseur is now passed, and the tissues thus surrounded are gradually divided. More recently $V_{\text {erneuil }}^{b}$ has recommended the opening into the rectum to be made with a fine point of Paquelin's cautery, instead of the trocar and cannula. Van Buren is of opinion that the entire section is

a Bull. de la Soc. Chirurg., October, 1872.

b International Medical Congress, Copenhagen, 1884. 
best made with a knife-shaped cautery, the charring of the edges protecting the wound from irritation of the fæces until granulation is established: he also avoids making it directly backwards, as he considers that the wound heals better when made a little to one side. Of all methods the knife is, I am satisfied, the best. With a probe-pointed bistoury the division can be more surely, expeditiously, and cleanly made than by any other means; and if it is confined to the middle line there need be no fear of bleeding. Any vessel which does spring can easily be ligatured; or, if there is general oozing, the wound may be plugged with an aseptic sponge, or, better still, with iodoform gauze, and the whole supported with a $T$-bandage. At first there will be a certain amount of incontinence, but as the wound heals the power of retaining fæces will gradually be regained. During the healing, frequent syringing with some antiseptic solution, preferably corrosive sublimate ( 1 in 2,000 ) should be employed; or, until granulation is established, continuous irrigation with warm solution may be resorted to.

The following two cases are good examples of this operation for the relief of non-malignant stricture :-

Case I.-A gentleman, aged twenty-three, consulted me in December, 1887. He had suffered from syphilis five years previously; he was pallid and emaciated, and complained of the greatest difficulty in defæcation, and had a continuous discharge of bloody mucus from the anus. Digital examination showed a ring of ulceration round the entire circumference of the gut, most marked in front, and immediately above the ulcerated surface a tight stricture was to be felt; the tip of the index finger would not pass into it; and it felt hard and rigid. Attempts to dilate with bougies gave rise to great irritation, and rather aggravated his condition. His abdomen became distended, and he had some vomiting. With the assistance of my colleague, Professor Bennett, and Dr. Browne (December 27, 1887), I divided the stricture posteriorly, the incision passing up into healthy tissue above, and extending through the anus to the tip of the coccyx and into the hollow of the sacrum. 'The relief to the symptoms of obstruction was immediate, and he made a good recovery. Defæcation now is 
normal; he only has a very slight amount of incontinence if he gets an attack of diarrhoea; the ulceration has quite healed, and there has been no recurrence of the stricture; he is quite strong, and looks thoroughly healthy.

CASE II.-Although rather at a loss to define the pathological condition in this case, the indications for linear proctotomy were obvious :-

Mrs. A., aged forty-seven years, recommended by Dr. Kenny, of Killeshandra, consulted me, February, 1886. Three years ago she noticed right breast becoming shrunken and nipple retracted, but she never had any pain in it; the left breast became similarly affected about eighteen months subsequently, and several nodules appeared over abdomen, which disappeared, leaving puckered subcutaneous cicatrices. Since then she suffered from ascites, and has been tapped six times; and for the past twelve months has had gradually increasing difficulty in getting the bowels to move. When I first saw her, her condition was as follows :-Appears well nourished, active, and healthy, has no pain, and only complains of discomfort when ascites becomes considerable. Breasts, nipples both quite retracted, the glands themselves feel somewhat nodular, but not hard; the skin is puckered and adherent, with what look like subcutaneous cicatrices-there is a similar condition in several places over the abdomen. The abdominal cavity contains a moderate quantity of ascitic fluid, but is not fully distended; the upper portion of the vagina is contracted and rigid, and the uterus immovable, but the surface smooth and free from nodules. A smooth stricture of the rectum exists $2 \frac{1}{2}$ inches above the anus. By the use of aperient medicine, and occasional passage of bougies, her condition was somewhat improved, but the contraction of the rectum gradually increased; and by the end of 1887 symptoms of intestinal obstruction became developed, with vomiting and great abdominal distension. Accordingly, as the obstruction was obviously tending to her death, I performed linear proctotomy, assisted by Drs. Kenny and Bennett, in January, 1888. The relief to the symptoms of obstruction was immediate and complete, and she gradually recovered, the ascites quite disappearing. She subsequently went, for the summer, to Bundoran, and died there, with symptoms of obstruction to the osophagus, having had no recurrence of the symptoms of intestinal obstruction. Unfortunately there was no opportunity of an autopsy in this most remarkable 
case. I have never met with a description of any case like hers; it certainly was not carcinoma, but appeared more to be a condition of disseminated cirrhosis of the various structures implicated, in some respects resembling the cheloid of Addison.

As examples of the removal of neoplasms by the aid of linear proctotomy the following are instructive :-

CASE III.-A man, aged sixty-three years, was admitted into Sir Patrick's Dun's Hospital, October, 1884, with piles, fissure, and history of obstruction in the rectum. Digital examination rerealed the presence of a tumour occupying the hollow of the sacrum, and compressing the rectum towards the bladder. As the finger could be passed above the upper limit of the growth, an attempt was made by linear proctotomy to remove the tumour, or, at any rate, to relieve the obstruction, the symptoms being so urgent that either this operation or colotomy was indicated. The incision was made in the usual manner, and, by means of the finger and scoop, the tumour, which proved to be in part a lympho-sarcoma, was broken down, and as much as possible removed, the obstruction being completely overcome. As hæmorrhage was free the wound was plugged, but unfortunately the patient got diffuse pelvic cellulitis, followed by peritonitis, and died on the sixth day.

CAse IV.-October 4, 1887, I saw, with Dr. Wright, of Dalkey, and Drs. Finny, O'Farrell, and Bennett, a gentleman, aged twentysix, who had been suffering for some months from blood-stained diarrhoa, with abundant discharge of intestinal mucus; he was extremely emaciated, blanched, and obviously his life was in very grave danger. Digital examination revealed a soft growth at the upper portion of the rectum, but so high up that its details could not be made out or its limits fixed. I advised that the only prospect of saving his life lay in the attempt to remove the mass by the aid of linear proctotomy, which was accordingly performed the next day. Immediately after the incision was made he became pulseless, and his respiration was very shallow, so that we had to resort to artificial respiration for a considerable time. By the facility afforded by the incision the growth could be easily explored; it was about the size of a florin, soft, and extremely vascular. In his enfeebled condition it was obvious that any attempt to remove it, if attended with further hæmorrhage, would be immediately fatal, so I destroyed it as completely as possible with the actual cautery. 


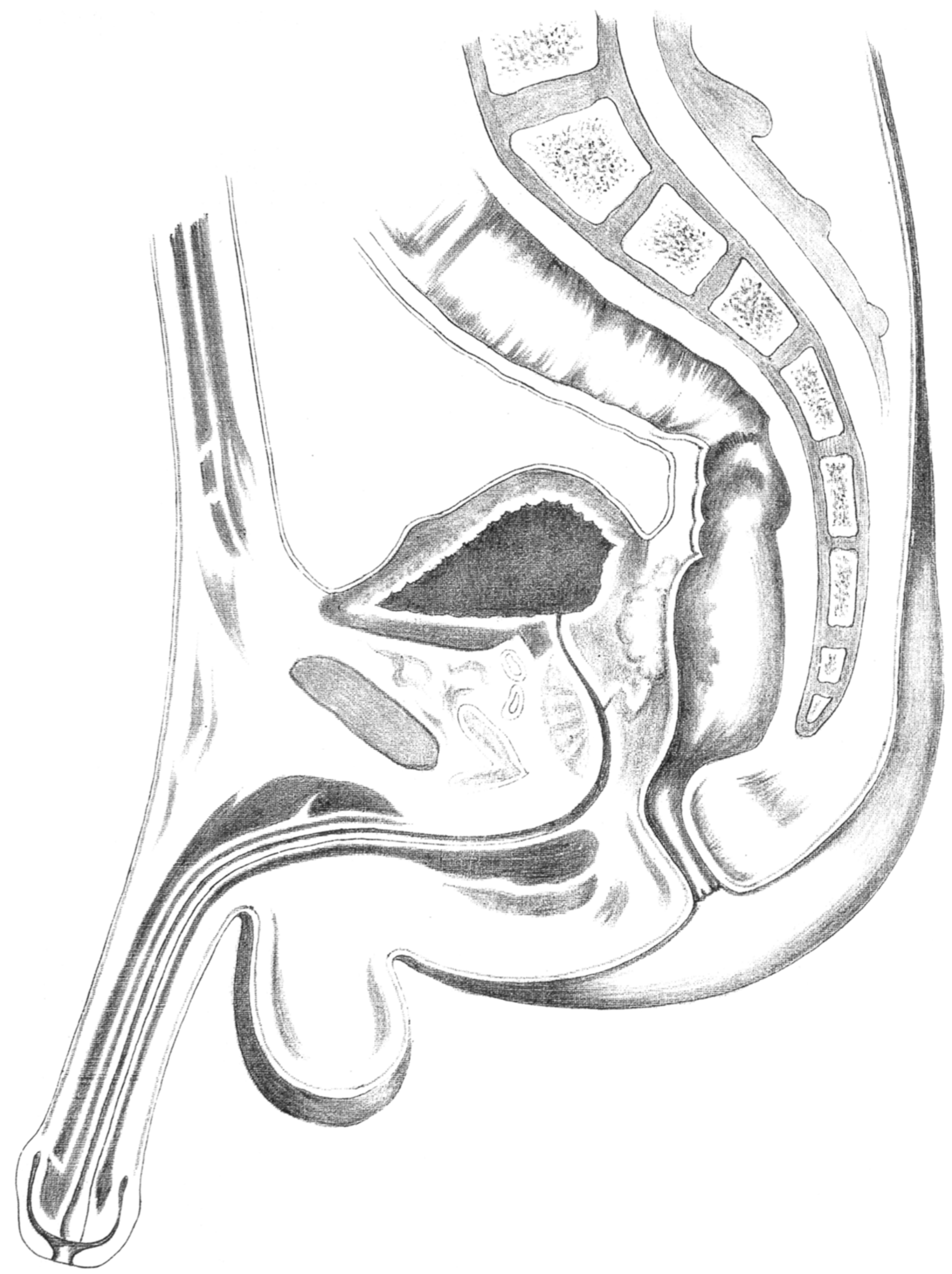

DIAGRAM OF VERTICAL SECTION OF THE MALE PELYIS. Drawn to Scale from the Average of three Frozen Sections. 
The patient made a slow but eventually perfect recovery, and a year subsequently was in good health, able to walk long distances; no incontinence of fæces, and no evidence whatever of recurrence.

The recommendation of Verneuil and Kelsey to treat obstruction due to malignant disease by linear proctotomy is not likely to be largely followed, and is, I believe, not good surgery. For, in the first place, if the disease is low enough down to be benefited by linear incision, it is probably better treated by excision; and even if the latter operation be contra-indicated by any other condition the advantages to be gained by diverting the fæces from a hopelessly cancerous rectum by means of a well-timed colotomy are now pretty generally admitted.

Linear proctotomy as a first step of amputation of the rectum is now, I think, practised by most surgeons, and in all cases in which I have operated for the past six years I have employed it. The advantages are-complete command over the rectum, easy performance of the further steps of the operation, and last, but not least, it provides a thoroughly efficient channel for drainage subsequently. The operation of linear proctotomy is one by no means devoid of danger; the incision necessarily penetrates into the loose areolar tissue of the pelvis, so that the risk of septic absorption is real-as was dernonstrated by one of the cases above recorded. This case, however, occurred several years ago, before the efficient antiseptic measures now employed had become evolved. But, on the other hand, when successful, it may be the means of saving many a valuable life. In order to appreciate the full advantage to be gained by this procedure it is necessary to refer to anatomical details, and for this purpose it is better to make use of frozen sections, as by this means a truer estimate can be found than by any other method of dissection. Through the kindness of Professor Cunningham I am enabled to produce a diagram, which is drawn accurately to scale and is the average of three vertical sections of the male pelvis. It will be seen that there is a very considerable amount of tissue between the anus and the tip of the coccyx-the Ano-coccygeal body of Symington. This body in the female is 
given by Ranney and Barbour as being relatively longer. From a glance at this diagram it will be seen that an incision through the ano-coccygeal body will open up the rectum well above the anal canal, where the gut is normally wide: and as syphilitic ulceration is usually situated in the pouch below the prostate gland, with its consequent stricture immediately above this point, it is obvious that not only is the strictured bowel completely divided, but the healthy intestine above opened up. As far as my experience goes, if the operation is thoroughly done and care taken to prevent contraction during healing, a permanent cure of rectal stricture may be looked for by this means. 